\title{
PROBES, POPULATIONS, SAMPLES, MEASUREMENTS AND RELATIONS IN STEREOLOGY
}

\author{
ROBERT T DEHOFF \\ Department of Materials Science and Engineering, University of Florida, Gainesville, FL 32611, USA \\ (Accepted November 4, 1999)
}

\begin{abstract}
This summary paper provides an overview of the content of stereology. The typical problem at hand centers around some three dimensional object that has an internal structure that determines its function, performance, or response. To understand and quantify the geometry of that structure it is necessary to probe it with geometric entities: points, lines, planes volumes, etc. Meaningful results are obtained only if the set of probes chosen for use in the assessment is drawn uniformly from the population of such probes for the structure as a whole. This requires an understanding of the population of each kind of probe. Interaction of the probes with the structure produce geometric events which are the focus of stereological measurements. In almost all applications the measurement that is made is a simple count of the number of these events. Rigorous application of these requirements for sample design produce unbiased estimates of geometric properties of features in the structure no matter how complex are the features or what their arrangement in space. It is this assumption-free characteristic of the methodology that makes it a powerful tool for characterizing the internal structure of three dimensional objects.
\end{abstract}

Keywords: probe populations, sampling, stereology.

\section{INTRODUCTION}

You have a problem. The glob of matter on the lab bench performs in interesting ways that you need to understand. You know in a qualitative way that how it performs depends upon its internal structure; you need to make that understanding quantitative. You know the glob has an internal structure because you have cut open globs like it, prepared them in the time honored way, and examined pieces; you have seen the structure. You know that the pieces that you have examined are just a sample of the structure of the glob, and that this sample is biased. It's biased because the structure of the glob is a three dimensional thing, and the samples you have seen are images in an eyepiece, or on a screen, or on the printed page. You can make measurements on these two dimensional images in your attempt to raise your understanding of how the glob works to a quantitative level. But how is that biased sample of two dimensional measurements related to the geometry of the real three dimensional structure of the glob?

Stereology, that's how.

Stereology is the methodology that provides meaningful quantitative descriptions of the geometry of real three dimensional glob structures from measurements that are made on two dimensional images sampled from the glob. The components of the stereological methodology are:
Features. The structure of the glob is composed of sets of specific features (particles, surfaces, interfaces, lines, edges, points, ...).

Properties. Each feature has one or more geometric properties which can be measured. The goal of the exercise is the quantitative estimation of these geometric properties.

Probes. The glob is sampled with a set of geometric probes - points, lines, planes, volumes-, on which observations and measurements are made.

Events. The probes interact with specific features in the structure to produce events to be observed (point probe lies in feature, line probe intersects boundary, plane probe transects particle, ...).

Measurements. Some attribute of each of the observed events of interest is measured. In most cases this is a simple count of the number of events observed.

Analysis. A mean and standard deviation of the distribution of measured values in the sample is computed. If the sample design is valid, the mean will provide an unbiased estimate for the expected value of the measurement for the population of probes.

Relationship. The fundamental equations of stereology relate the expected values of specific measurements on an appropriate probe set to a 
corresponding geometric property in the three dimensional glob.

Design. Each kind of probe has a specific population in the space occupied by the glob. The choice of the subset of probes in this population to be examined for measurement constitutes the design of the stereological experiment.

The design of a stereological experiment, by which is meant the set of choices that are made for the selection of orientations and positions of the fields to be observed and measured, is the most difficult and most important component of practical stereology. An improper design could lead to the expenditure of a great deal of effort to produce very precise estimates of wrong numbers.

\section{FEATURES}

A microstructure is a space filling arrangement of features in three dimensional space. The features themselves may be volumes, surfaces, lines, or points. Plane sections through the structure reveal these features respectively as areas (from volume features), lines (from surface features, or points (from line features); point features in three dimensions are not directly observable on a section.

Most microstructures can be visualized as a subdivision of three dimensional space into cells, - a tessellation. In the simplest case there is only one kind of cell, say the $\alpha$ type. Cell boundaries may be labeled " $\alpha \alpha$ " boundaries because there is an $\alpha$ side on each side of the boundary. Triple lines in this cell structure result from the incidence of three $\alpha$ cells: $\alpha \alpha \alpha$ triple lines. Vertices in the cell structure arise from four cells meeting in space to form $\alpha \alpha \alpha \alpha$ quadruple points. These features are listed in column 2 of Table 1.

The possible features that may occur in two phase $(\alpha+\beta)$ structures are listed in column 3. Some of these features may be absent in a two phase structure; for example, if the $\beta$ features have no internal boundaries $(\beta \beta)$ then all entities in the feature list that have two $\beta$ 's in their description will not appear.

\section{PROPERTIES}

Each of the features in these lists has associated geometric properties, columns 4 and 5. Most familiar of these properties are the measures of extent, volume, surface area and line length, which report the total quantity of that kind of feature in the structure. The total curvature $\mathrm{M}_{\mathrm{V}}$ involves the concepts of curvature at a point on a surface and its integral over the surface (DeHoff and Rhines, 1968). The topological properties, number, and connectivity, which is the number of cuts that may be made on a feature without severing it into two parts, provide rudimentary information about the skeleton on which the structure is built. The spherical image (DeHoff and Rhines, 1968), $\Omega_{\mathrm{V}}$, provides components of the topological properties.

\section{All of the properties on this list share two attributes:}

1. Each has rigorous unambiguous meaning for real features of arbitrary geometry.

2. Each is accessible to unbiased stereological estimation.

These attributes add to the power of the methodology of stereology.

\section{PROBES}

Knowledge of the structure of the glob is obtained by sampling it with geometric probes, typically point, line, plane or volume probes. In practice this is usually done by cutting the sample (a plane probe) and superimposing upon the two dimensional image thus exposed a grid of lines or points. The volume probe, called the disector, is formed from the volume contained between two sectioning planes which are close enough together to infer geometric events that occur in the volume between them.

Each of these geometric probes has its population in the three dimensional space that contains the glob. In order to devise appropriate designs for sampling these probes in stereological experiments it is crucial to understand the nature of these probe populations.

Point Probes. The population of point probes is simply the set of points in three dimensional space.

Line Probes. A line in space has two kinds of attributes: orientation or directions, and for a given direction, position. An orientation is represented by a point on a unit sphere (longitude and latitude). The population of all possible orientations is the population of points on the unit sphere. For a given orientation, the population of line positions may be visualized by constructing a plane that is perpendicular to that orientation. A specific line with that orientation passes through a point on that plane. Thus, the population of line positions for a given orientation is the population of points on the plane perpendicular to that orientation.

Plane Probes. A plane in space also has attributes of orientation and position. The orientation of a plane is visualized the direction of a line that is perpendicular to the plane (the normal to the plane). The population of plane orientations also corresponds to the population of points on the unit sphere. For a given orientation, the population of planes may be visualized by constructing 
a line in that orientation. A specific plane with the given orientation passes through a point on that line. Thus, the population of plane positions for a given orientation is the population of points along a line that has that orientation.

Disector Probes (Sterio, 1984). A disector is a small box that samples the volume of the glob. It is contained between two planes a known small distance $h$ apart and the area, $\mathrm{A}_{0}$, of the field on one of the planes. A disector may be uniquely located by a characteristic point, e.g. the upper left back corner of the box. Thus the population of disectors is identical with the population of points in three dimensional space.

Sweeping Plane Probe (DeHoff and Rhines, 1968). Closely related to the disector probe, the sweeping plane probe visualizes a plane that is swept through a sample of the volume of the structure. The population of sweeping plane probes is the population of orientations in space which is given by the set of points on the unit sphere.

\section{EVENTS}

Each kind of probe interacts with a specific class of features in the microstructure to produce events which are the focus of the subsequent analysis. These combinations of probes and events are laid out in columns 1 and 2 of Table 2 .

Point Probes. The population of point probes is sampled by selecting a plane probe and a field on that plane. A grid of points is superimposed on the structure in that field. The structure may consist of a number of different classes of volume features, $\alpha, \beta, \gamma, \ldots$ Focus on one class for analysis, e.g., $\beta$. The event of interest is that a point lies within $\beta$ features.

Line Probes. The lines in the grid described above may be used to sample the population of line probes, or special line grids may be constructed. The structure may contain different classes of surfaces, $\alpha \alpha, \alpha \beta, \beta \beta, \ldots$ Focus on one class of surfaces in the analysis, say, $\alpha \beta$. The events of interest are the intersections of a line probes in the sample with $\alpha \beta$ boundaries.

Plane Probes. The section produced through the sample be cutting it and preparing the exposed surface for observation is a plane probe. If the three dimensional feature class under study is a set of lines, e.g., the $\alpha \alpha \beta$ triple lines in the structure, the events of interest are the intersections of the plane probe with these lineal features. These events appear as points on the plane probe, e.g., $\alpha \alpha \beta$ triple points for the feature set above. If the feature set of interest is a collection of particles, e.g., of the $\beta$ class of features, then the event is the intersection of the plane probe with particles in this set. These appear as two dimensional transects on the plane probe.

Disector Probe. The disector probe is formed by producing two plane probes a known small distance apart. One plane is designated the reference plane, and the other the look up plane. An area is delineated on the reference plane, which, together with the distance between the planes, establishes the volume of the probe. The events of interest are particle sections that appear on the reference plane, but are not present in the look up plane. The disector probe may be produced physically, by preparing the pair of planes for observation, or, in transparent globs, optically, by vertically scanning the focal plane of a confocal microscope (Gundersen, 1986).

Sweeping Plane Probe. The sweeping plane probe requires a disector, a collection of serial sections, or, for a transparent glob, a confocal microscope. A plane is swept through the structure contained in the volume between the prepared surfaces. The events of interest are the formation of tangents with the surfaces bounding the particles in the structure.

\section{MEASUREMENTS}

In each of the interactions between probes and structural elements described above, one may imagine marking the events as they are encountered. The measurements of stereology simply involve counting these marks, and normalizing that number by dividing by the size of the probe used. Important measurements are listed in Column 3 of Table 2. Symbols for the corresponding normalized versions of these counts are given in Column 4.

Point Probe: If $\mathrm{P}_{0}$ is the total number of points in the grid used to sample a given field and $\mathrm{P}^{\beta}$ points are marked because they lie within the $\beta$ phase, then the ratio, $P_{P}^{\beta}=P^{\beta} / P_{0}$ is the normalized "point fraction" for the $\beta$ features the field.

Line Probe. The total length of lines probed in the field is measured by calibrating the magnification and found to be some number $\mathrm{L}_{0}$. Intersections with the $\alpha \beta$ interface (for example) are marked and counted; let that number be $\mathrm{P}^{\alpha \beta}$. The normalized "line intersection count" for $\alpha \beta$ surfaces in the field is $P_{L}^{\alpha \beta}=P^{\alpha \beta} / L_{0}$. 
Table 1. Feature classes that may exist in one and two phase microstructures and their geometric properties.

\begin{tabular}{l|c|c|l|l}
\hline Feature Class & $\begin{array}{c}\text { Single } \\
\text { Phase }(\alpha)\end{array}$ & $\begin{array}{c}\text { Two Phase } \\
(\alpha+\beta)\end{array}$ & \multicolumn{1}{c}{$\begin{array}{c}\text { Geometric } \\
\text { Property }\end{array}$} & Symbol \\
\hline Volumes & $\alpha$ & $\alpha, \beta$ & $\begin{array}{l}\text { Volume Fraction } \\
\text { Number } \\
\text { Connectivity }\end{array}$ & $\begin{array}{l}\mathrm{V}_{\mathrm{V}} \\
\mathrm{N}_{\mathrm{V}} \\
\mathrm{C}_{\mathrm{V}}\end{array}$ \\
\hline Surfaces & $\alpha \alpha$ & $\alpha \alpha, \alpha \beta, \beta \beta$ & $\begin{array}{l}\text { Surface Area } \\
\text { Total Curvature } \\
\text { Spherical Image }\end{array}$ & $\begin{array}{l}\mathrm{S}_{\mathrm{V}} \\
\mathrm{M}_{\mathrm{V}} \\
\Omega_{\mathrm{V}}\end{array}$ \\
\hline Triple Lines & $\alpha \alpha \alpha$ & $\alpha \alpha \alpha, \alpha \alpha \beta, \alpha \beta \beta, \beta \beta \beta$ & Line Length & $\mathrm{L}_{\mathrm{V}}$ \\
\hline Quadruple Points & $\alpha \alpha \alpha \alpha$ & $\begin{array}{c}\alpha \alpha \alpha \alpha, \alpha \alpha \alpha \beta \\
\alpha \alpha \beta \beta, \alpha \beta \beta \beta, \beta \beta \beta \beta\end{array}$ & Number & $\mathrm{N}_{\mathrm{V}}$ \\
\hline $\begin{array}{l}\text { Total Number of } \\
\text { Classes }\end{array}$ & 4 & 14 & & \\
\hline
\end{tabular}

Table 2. Geometric probes, their associated events and measurements.

\begin{tabular}{l|l|l|l}
\hline Probe & Event & Measurement & Symbol \\
\hline Point & Point lies in feature & Count points & $\mathrm{P}_{\mathrm{P}}$ \\
Line & Line intersects feature surface & Count intersections & $\mathrm{P}_{\mathrm{L}}$ \\
Plane & Plane intersects space curve & Count intersections & $\mathrm{Q}_{\mathrm{A}}$ \\
& Plane transects particle & Count transects & $\mathrm{N}_{\mathrm{A}}$ \\
Disector & Feature ends in the volume & Count ends & $\mathrm{Q}_{\mathrm{V}}$ \\
Sweeping & Sweeping plane forms tangent in & Count tangents & $\mathrm{T}_{\mathrm{V}}$ \\
Plane & volume & & \\
\hline
\end{tabular}

Plane Probe. A frame is delineated on the field of view and its area $\mathrm{A}_{0}$ measured. The points of intersection of lineal features, e.g., the $\alpha \alpha \beta$ triple line, are marked and counted: let $\mathrm{P}^{\alpha \alpha \beta}$ be the number of intersection points in the frame. The normalized "area point count" for $\alpha \alpha \beta$ triple lines in this field is $Q_{A}^{\alpha \alpha \beta}=Q^{\alpha \alpha \beta} / A_{0}$.

If the features of interest are particles of the $\beta$ feature set, then sections through these particles will occupy some distribution of areas on the plane probe. Counting the number of two dimensional $\beta$ sections presents a problem: because they have a finite size; some will intersect the boundary of the frame. An unbiased count of these events can be obtained by using an "unbiased frame" (Gundersen, 1977). For simple structures, this count can be made by excluding particles that intersect two sides of the frame, and including those that lies inside the frame and those that intersect the other two sides. If $\mathrm{N}^{\beta}$ is the number of particle sections thus counted, the normalized "feature count" is $N_{A}^{\beta}=N^{\beta} / A_{0}$.
Disector Probe. On a given field the frame area is calibrated to be $\mathrm{A}_{0}$, and the distance between the pair of plane probes is $h$. The volume of the disector sample is thus $\mathrm{V}_{0}=\mathrm{A}_{0} \mathrm{~h}$. The unbiased frame strategy described above is applied to the reference plane to identify the set of $\beta$ particle sections to be examined. The look up plane is scanned to determine which of these particles do not extend to that plane. It is inferred that these particles ended within the volume $V_{0}$. Let the number of "particle ends" contained in the sample volume be $\mathrm{Q}^{\beta}$. The normalized particle count for the sample is $Q_{V}^{\beta}=N^{\beta} / V_{0}$.

Sweeping Plane Probe. The sample is a disector with known volume $\mathrm{V}_{0}$, a more comprehensive collection of serial sections, or a larger volume to be scanned with a confocal microscope. Tangent events may occur with three classes of surface elements: convex $(++)$, concave (- -), and saddle surface elements, $(+-)$. These tangents are counted separately, giving three normalized counts defined by

$T_{V_{++}}=T_{++} / V_{0} ; T_{V_{--}}=T_{--} / V_{0} ; T_{V_{+-}}=T_{+-} / V_{0}$. 


\section{ANALYSIS}

The analysis of the measurements for each of the probe/event counts is essentially the same. Compute the mean value from the distribution of measured counts on the collection of fields included in the experiment. Compute the standard deviation of this sample from the population of probes. Use the standard deviation to compute the standard deviation of the mean of the population of samples of size $\mathrm{n}$. Compute confidence intervals for the experimental estimate as the mean value \pm two standard deviations of the mean (for a $95 \%$ confidence interval). This reports the precision of the estimate. The confidence interval can in principle be made arbitrarily small by increasing the number of fields measured in the experiment.

The mean value obtained from the sample is used to estimate the expected value for the population of probes used in the measurement. It is crucial that this estimate be unbiased. Unbiasedness can only be achieved through a knowledgeable design of the sample. If the sample design gives a biased result, then a significant effort may be expended to provide a very precise estimate of a wrong number.

Recent experimental and theoretical studies have shown that the design of the experiment can effect significantly the standard deviation of the set of sample readings (Gundersen and Jensen, 1987). For example, the standard deviation of a sample of a set of probes chosen randomly (i.e., in a process involving a random number generator) is significantly larger than the standard deviation for a sample design described as a "systematic random" sample. Thus, application of the random systematic sample design significantly decreases the number of measurements that must be made to achieve a given level of precision.

\section{RELATIONSHIPS}

The stereological relationship connect the measurements described in Column 4 of Table 2 with the properties defined in Column 5 of Table 1. Although the mathematics underlying these relationships is sophisticated and multifaceted, involving differential geometry, integral geometry, topology and measure theory, the relationships themselves are surprisingly simple. Each of these relations is an expected value theorem. The expected value of a statistic is the value that would be obtained if the entire population were included in the sample from which the statistic is obtained. Thus, the relationship $\left\langle P_{P}\right\rangle=V_{V}$ means, "The expected value of the point fraction is equal to the volume fraction." That is, the value of $\mathrm{P}_{\mathrm{P}}$ for a particular feature set for the population of points in three dimensions is the volume fraction occupied by that feature set.

In each of these relationships the expected value of the measurement applies to the population of whatever probe (points, lines, planes, disectors, sweeping planes) is used in the measurement. These relationships are summarized in Table 3 (DeHoff and Rhines, 1968; Underwood, 1970; Howard and Reed, 1998).

\section{DESIGN}

The fundamental stereological relations presented in Column 3 of Table 3 have been described as expected value relationships. The left side of each of these equations is the expected value of a statistic (Column 4 of Table 2) which is a normalized count of the number of events (Column 2 of Table 2) that result from the interaction of a specific probe (Column 1 of Table 2) with features in the microstructure. The right side is a geometric property of the three dimensional microstructure (Column 5, Table 1), the goal of the exercise. The equations are valid if the entire population of probes in three dimensional space is included in the measurement.

In practice a small sample of this population is selected for examination and measurement. The aim of sample design in stereology is to provide a set of probes for a measurement that yield a sample mean that is an unbiased estimate of the expected value of that parameter for the population. In this context "design" means making the right choices in deciding

1. What positions and orientations will be used to slice the sample and reveal its structure;

2. On this set of slices, what positional distribution of fields of view will be chosen for observation;

3. The design of the grid of lines or points to be samples of the population of point probes or line probes;

4. How many fields will be included in the measurement.

In order for the sample mean to be an unbiased estimator of the population mean it is necessary to choose the set of sample probes uniformly from the population of probes. In order to do this it is essential to understand the geometry of the population of each class of probe as spelled out in the section titled "Probes" above. 
Table 3. The stereological relationships.

\begin{tabular}{l|l|l|l}
\hline Probe & Measurement & Relationship & Property \\
\hline Point & Point count & $<\mathrm{P}_{\mathrm{P}}>=\mathrm{V}_{\mathrm{V}}$ & Volume fraction \\
Line & Line intersection count & $<\mathrm{P}_{\mathrm{L}}>=1 / 2 \mathrm{~S}_{\mathrm{V}}$ & Surface area \\
Plane & Area point count & $<\mathrm{Q}_{\mathrm{A}}>=1 / 2 \mathrm{~L}_{\mathrm{V}}$ & Line length \\
Plane & Feature count & $<\mathrm{N}_{\mathrm{A}}>=\mathrm{M}_{\mathrm{V}} / 2 \pi$ & Total curvature \\
Disector & Disector feature count & $<\mathrm{Q}_{\mathrm{V}}>=\mathrm{N}_{\mathrm{V}}$ & Feature number \\
Sweeping Plane & Tangent count & $<\mathrm{T}_{\mathrm{V}}>=\Omega_{\mathrm{V}}$ & Spherical image \\
\hline
\end{tabular}

Point Probes. The sample of points must be drawn uniformly from the population of points in three dimensions. This implies that the sections taken should be uniformly spaced through the sample and that the fields chosen for observation on these sections must be uniformly distributed over the section areas. The number of fields observed in a typical experiment is in the range of 10 to 40 , depending upon the precision required and the number of events that occur in a typical field. If for example 20 fields are assessed one might produce five slices with four fields on each slice. A few fields on a number of sections sample the structure more uniformly than a lot of fields on a few sections.

Line Probes. The set of lines included in a sample of the population of line probes in space must have directions that are drawn uniformly from the set of points on the unit sphere, and uniformly with respect to position in the three dimensional sample. This would at first appear to be an impossible task in sample design. Fortunately there exists a sample design strategy for this case, called the method of vertical sections, which automatically produces such a sample of line probes.

Plane Probes. The set of planes in the sample of the population of planes in space must have directions that uniformly sample the set of orientations on the unit sphere and the set of positions along each direction. Producing a uniform sample from the population of plane probes is perhaps the most difficult of sample design problems. It is possible to devise a design strategy which also achieves this goal automatically, but, as will become clear later, this strategy is not particularly practical.

Disector Probes. Because the disector sample is a volume, it does not share the attribute of orientation with lines and plane probes. Disectors only have position, so it is only necessary to sample positions in the glob uniformly. In cases where the entire glob is sliced on a microtome, it is only necessary to choose a small number of sections uniformly distributed from the set of slices as reference planes, and then in each case choose a second slice a short distance away as the look up plane. As in the case of the point probe sample, it is best to examine a few fields uniformly distributed on each reference plane in combination with a number of reference plane sections, rather than examine a lot of fields on a few reference planes.

Sweeping Tangent Probe. Because this probe is used to estimate topological properties (number, connectivity from the spherical image), and topological properties do not possess the attribute of orientation, it is only necessary to sweep the plane through the sample volume in one direction. The set of sample volumes examined is a set of disectors, and the rules for obtaining a uniform sample from the population of disectors are described in the last section.

\section{SPECIAL SAMPLING STRATEGIES}

The "random systematic sampling" strategy is used pervasively in stereology in producing as set of samples of probes for observation (Howard and Reed, 1998). Imagine the glob is sliced into 50 slabs. Preliminary work has established the number of fields that should be examined stereologically. Suppose 30 fields need to be included in the measurement. An efficient distribution might suggest that examination of 5 plane probes with an average of 6 fields on each will sample the three dimensional glob uniformly. Thus, about 1 out of 10 slabs will need to be prepared for observation. Choose a random number between 1 and 10. Suppose this comes out as 7. Prepare the following slabs for inspection: 7, 17, 27, 37, 47. This sample of the slabs is random and systematic.

As a second sampling stage examine one of these prepared slabs, say number 27. From the dimensions of the slab and the magnification to be used a total of perhaps 100 separate fields might potentially be constructed on this surface. Imagine labeling these positions from 1 to 100 . To choose the six that will be inspected, divide 100 by 6 to obtain approximately 16 . Choose a random number between 1 and 16; suppose it 
comes out to be 7 . Using the stage controls, locate the positions of field number $7,(7+16)=23,(23+16)=$ $39,55,71,87$. Apply this strategy to the other five plane probes. The result is a random systematic distribution of fields that sample the three dimensional glob uniformly as the stereological relations require.

To obtain a uniform sample of line probes in order to estimate the surface area apply the "method of vertical sections" (Baddeley et al., 1986). This requires that a reference direction be chosen for the glob, and that knowledge of this reference direction be maintained in every field examined. Imagine the glob is sliced into sticks like french fries from a potato. The long edge of each stick is the "vertical" direction. Use a random systematic strategy to select the small number of sticks that will be included for evaluation. Mount the sticks thus selected for fixing and preparation, rotating them randomly and uniformly about their long axes. A single plane section through this fixed aggregate of samples uniformly samples positions of the fields, and the longitudinal component of the orientation about the vertical axis. A random systematic sampling strategy is again used to select the fields on this section that will be evaluated. It remains to sample the direction of line probes in the latitude variable on the unit sphere. This can be accomplished by constructing a curved line probe in which the elements of length point in directions that vary from vertical to horizontal in a way that gives appropriate weight to the length of probe in each incremental direction. It has been shown that such a curve is the cycloid. This strategy automatically generates a uniform sample of the populations of positions and orientations of lines in three dimensional space. Producing such a sample requires little additional effort beyond that for normal preparation of a microstructure for observation; it is mostly a matter of planning ahead.

A similar strategy called the method of vertical slices (Gokhale, 1990) has been devised for sampling the population of orientations of plane probes in transparent structures that may be viewed as a projection image obtained from a transparent slice. In this case a cycloid line on the projected image is the projection of a cycloid surface in the sample volume. If the cycloid is properly oriented relative to the vertical direction chosen for the glob, then the population of plane orientations in the latitude variable is uniformly represented on this cycloid surface.

For opaque globs one can envision an ultimate sampling strategy. Dice the glob into cubes. Use a random systematic sampling strategy to select the cubes for preparation. This guarantees uniformity with respect to position. Mount the cubes by placing them on a plastic mold that has an array of variously oriented cube shaped dimples that are designed to sample the sphere of orientations uniformly. Cover with the mounting medium and cure. A section of this aggregate of samples will automatically produce a uniform isotropic sample of plane probes. Grids placed on these planes will have lines that are also uniformly and isotropically drawn from the population of lines. Points on these grids will be uniformly drawn from the population of points. A set of fields selected by a random systematic procedure will satisfy all of the sampling requirements for all of the measurements of stereology except those that require the disector.

\section{SUMMARY}

Stereology is a powerful tool for characterizing the geometry of real, three dimensional microstructures. The structure must be probed with points, lines, planes or volumes. Interactions of the probes with features in the microstructure produce events that are counted and these counts averaged over the structure. With a firm knowledge of the geometric structure of the populations of these probes, samples can be designed for which the mean values are unbiased estimates of their expected values for the population of probes. This essential element permits application of the fundamental relations of stereology which relate these expected values to the geometric properties of the three dimensional structure which they measure. The power of the method derives from the attribute that the resulting measures have unambiguous meaning for structures of arbitrary complexity.

The subject of this review paper was presented as overview lectures (DeHoff, 1999) at the $\mathrm{X}^{\text {th }}$ International Congress for Stereology, Melbourne, Australia, 1-4 November 1999.

\section{REFERENCES}

Baddeley AJ, Gundersen HJG and Cruz-Orive LM (1986). Estimation of surface area from vertical sections. J Microsc 142:259-76.

Braendgaard H, Evans SM, Howard CV and Gundersen HJG (1990). The total number of neurons in the human neocortex unbiasedly estimated using optical disectors. J Microsc 157:285-304.

DeHoff RT and Rhines FN (1968). Quantitative Microscopy. Ch 10. New York: McGraw-Hill.

Gokhale AM (1990). Unbiased estimation of curve length in 3-D using vertical slices. J Microsc 159:133-43. 
Gundersen HJG (1977). Notes on the estimation of numerical density of arbitrary profiles; the edge effect. J Microsc 111:219-23.

Gundersen HJG (1986). Stereology of arbitrary particles. A review of unbiased number and size estimators and the presentation of some new ones, in memory of William R. Thompson. J Microsc 148:3-45.
Gundersen HJG and Jensen EB (1987). The efficiency of systematic sampling in stereology and its prediction. $\mathrm{J}$ Microsc 147:229-63.

Sterio DC (1984). The unbiased estimation of number and sizes of arbitrary particles using the disector. J Microsc 134:127-36.

Underwood EE (1970). Quantitative Stereology, Addison Wesley, Reading MA. 